\title{
Period-Luminosity-Metal Abundance Relations for Population II Variable Stars
}

\author{
James M. Nemec ${ }^{1,2}$ \& Thomas E. Lutz ${ }^{2}$ \\ ${ }^{1}$ Astronomy Department, University of Washington, Seattle WA 98195 USA \\ ${ }^{2}$ Program in Astronomy, Washington State University, Pullman WA 99164 USA
}

\begin{abstract}
New period-luminosity-metallicity $(P-L$ - $[\mathrm{Fe} / \mathrm{H}])$ relationships for Pop. II Cepheids, RR Lyrae stars, anomalous Cepheids and SX Phe (variable-blue straggler) stars are presented. These were computed by fitting regression lines to observed pulsation periods and mean $B, V, K$ magnitudes for over 1200 stars in 40 stellar systems. The stars were assumed to be pulsating in either the fundamental ( $F$ ) or first-overtone $(H)$ modes (excluding double-mode and other multi-periodic variables). Eight $P-L-[\mathrm{Fe} / \mathrm{H}]$ relationships (one for each of the two pulsation modes for the four kinds of stars) were simultaneously fitted for each filter. After accounting for the metal abundance differences, the slopes of the $P-L$ relations were tested for departures from equality. The results are consistent with the assumption that, for each kind of star, the relations for the $\mathrm{F}$ and $\mathrm{H}$ stars are vertically offset, with a family of lines corresponding to the different metallicities. In the case of the globular cluster Cepheids, the available $B, V$ data support Arp's 1955 contention that the Cepheids are oscillating in the $\mathrm{F}$ and $\mathrm{H}$ modes; moreover, the majority of the short-period Cepheids (BL Her stars) appear to be first-overtone pulsators, while most of the Cepheids with periods between 10 and 30 days (W Vir stars) appear to be fundamental-mode pulsators. For the RR Lyrae stars, the slopes of the $P-L$ - $[\mathrm{Fe} / \mathrm{H}]$ relations in $B, V$ and $K$ show a clear trend with filter type, namely, the absolute values of the slopes increase from $B$ to $K$. Finally, for the SX Phe stars the differences between the $P-L-[\mathrm{Fe} / \mathrm{H}]$ relations in $B$ and $V$ for the $\mathrm{F}$ and $\mathrm{H}$ stars are found to be consistent with the known period-ratio for the double-mode star SX Phe.
\end{abstract}

\section{Introduction}

Several kinds of variable stars occupy the Pop. II Cepheid instability strip: Pop. II Cepheids (P2Cs), anomalous Cepheids (ACs), RR Lyrae stars and SX Phe (variable blue straggler) stars. By far, most of these are RR Lyrae stars (see Sawyer Hogg 1973). All four kinds of stars are also found throughout our Galaxy's halo and nuclear bulge, in nearby dwarf galaxies, and in other more distant galaxies. Since the stars appear to obey $P-L$ relations which can be used to derive distances, it is surprising that, with the exception of the RR Lyrae stars, Pop. II variables are rarely used as distance indicators. This is in part because there are only a few P2Cs, ACs or 
SX Phe stars in any given cluster, and thus determination of the $P$ - $L$ relations is very uncertain. In addition much of the available photometry is limited to photographic $B$ magnitudes, and there is a dependence of the luminosity level of the RR Lyrae stars on $[\mathrm{Fe} / \mathrm{H}]$ and evolutionary state.

The aim of the present paper is to show that by analyzing all four kinds of Pop. II variables simultaneously, and by taking into account reddening corrections, metallicity differences, and the evolutionary states of the $R R$ Lyrae stars, it is possible to derive a consistent set of $P-L-[\mathrm{Fe} / \mathrm{H}]$ relations for the $B, V$ and $K$ passbands, from which more reliable distance estimates for globular clusters and nearby dwarf galaxies can be obtained. The estimated $P-L-[\mathrm{Fe} / \mathrm{H}]$ relations are obtained by a linear least squares regression analysis with indicator variables, or equivalently, an analysis of covariance (see §2). In general, this method can be used to carry out a simultaneous analysis of as many different types of stars, with different pulsation modes, in different clusters, and through various filter types, as the data allow. Estimated uncertainties for the model parameters are also calculated. This method of pooling the data has not previously been used to derive $P-L-[\mathrm{Fe} / \mathrm{H}]$ relations, and has potential for improving other distance estimation methods, e.g., Tully-Fisher, Faber-Jackson, etc.

To illustrate the power of the method, the mean photometric $B$ magnitudes of the pulsating variable stars in many globular clusters and dwarf galaxies are used in $\S 3$ to calibrate the $B$-passband $P-L-[\mathrm{Fe} / \mathrm{H}]$ relations for the four kinds of stars. In $\S \S 4-7$ we discuss the results of a simultaneous multi-wavelength analysis for the RR Lyrae stars, the Pop. II Cepheids, the anomalous Cepheids and the SX Phe stars.

\section{Methodology}

Each of the eight types of stars is assumed to follow a linear absolute magnitudeperiod-metallicity relation in each passband. For observations through the $B$ filter,

$$
M_{B i j k}=a_{B j}+b_{B j} \log P_{i j k}+c_{B}[\mathrm{Fe} / \mathrm{H}]_{k}+\epsilon_{B i j k} .
$$

The absolute $B$ magnitude and the corresponding pulsation period (log base 10) are denoted $M_{B i j k}$ and $\log P_{i j k}$, respectively, where the subscripts $i, j$ and $k$ represent the 'star', 'type' and 'cluster'. For the cases considered here, the type index $(j=1,2, \ldots, 8)$ identifies the four kinds of stars and the two pulsation modes. The quantity $[\mathrm{Fe} / \mathrm{H}]_{k}$ represents the mean metal abundance of the stars in an individual stellar cluster $(k)$ or system. Of course, there will always be some unexplained variability due to random measurement error, represented by the term $\epsilon_{B i j k}$ in the model. The apparent $B$ magnitude is given by

$$
m_{B i j k}=a_{B j}+b_{B j} \log P_{i j k}+c_{B}[\mathrm{Fe} / \mathrm{H}]_{k}+d_{k}+A_{B k}+\epsilon_{B i j k},
$$

where we have transformed from absolute to apparent magnitude by adding to both sides of the equation the true distance modulus of the cluster, $d_{k}$, and the interstellar extinction in $B$ for cluster $k, A_{B k}$. The latter is equal to $R_{B} \mathrm{E}_{k}(B-V)$, where $R_{B}$ is the ratio of total to selective extinction for the $B$ passband, and $\mathrm{E}_{k}(B-V)$ is the 
$B-V$ reddening for cluster $k$. Similar equations could be written for other filters. We will be discussing, in addition to the $B$ passband, the $V$ and $K$ passbands.

The coefficients $a_{B j}, b_{B j}, d_{k}$ and $\epsilon_{B i j k}$ were estimated by first removing the effects of differences in the metal abundances and reddenings to obtain de-reddened, metallicity-corrected magnitudes:

$$
m_{B i j k}^{\prime}=m_{B i j k}-c_{B}[\mathrm{Fe} / \mathrm{H}]_{k}-A_{B}
$$

These 'corrected' magnitudes were then fitted to the model

$$
m_{B i j k}^{\prime}=a_{B j}+b_{B j} \log P_{i j k}+d_{k}+\epsilon_{B i j k}
$$

and the coefficients were estimated by ordinary unweighted least squares. The calculations were made using PROC GLM in the SAS statistical package.

To estimate the true distance moduli, values for the metal abundances and extinction coefficients were assumed. For the three filters, the metallicity coefficients were set equal to the values $c_{B}=0.35, c_{V}=0.32$ and $c_{K}=0.06$ (see Zinn 1985b; Longmore et al. 1990, hereafter L90; and Longmore 1993) for all four kinds of variable stars (P2Cs, RR Lyraes, ACs and SX Phe stars) - this assumption should be closely examined when more information becomes available. The interstellar extinction coefficients were assumed to be the standard values $R_{B}=4.1, R_{V}=3.1$ and $R_{K}=0.35$, for all but three of the program clusters, NGC 3201, NGC 6121(=M4) and N6171(=M107). These systems are known for their high and variable star-to-star extinctions, and, in the case of M4 and M107 there is also the possibility of abnormal ratios of total to selective extinction. To correct NGC 3201 the Cacciari (1984) internal extinction values were adopted, and for M4 and M107 we used $R_{V}=3.8$ rather than 3.1 (as recommended by $\mathrm{L} 90)$.

Student's $t$-test was used to test whether the lines for the fundamental and firstovertone pulsators are parallel for each filter. Failure to find a significant difference was taken to mean that the first-overtone and fundamental mode stars can be combined into a single line, or equivalently, the first-overtone pulsators can have their periods "fundamentalized" and then combined with the fundamental mode pulsators to form a single line (as L90 and other before them have assumed without testing). For the RR Lyrae stars tests were also made to see whether there was a vertical separation at a given period between the $a b$ - and $c$-type lines for the $B$ and $V P-L$ relations. Finally we tested whether the slopes of the $P-L$ lines were zero for each mode. This test is of interest only for the $B$ and $V$ regressions for the RR Lyrae stars since it is common practice to draw a horizontal line through the RR Lyrae stars in a cluster (i.e., assume a zero slope) and then to make a vertical shift to the RR Lyrae stars in another stellar system thereby establishing the distance of the latter.

The strength of our analysis method is that any number of different wavelength intervals, different types of stars, different clusters, etc., can be analyzed using this method. The distance scale is determined using not just the RR Lyrae stars but all of the available instability strip variables. The derived $P-L-[\mathrm{Fe} / \mathrm{H}]$ relations are 
estimated in a consistent manner, and estimates of the uncertainties in the slopes, zero points, and differences in distance moduli, can be derived. The method also has the advantage that one can determine the slope for each $P-L-[\mathrm{Fe} / \mathrm{H}]$ relation using only a small number of stars of each type in any one star system. Thus a substantially larger number of stars is available for examining each $P-L$ relation than would normally be available.

Finally, a comment about notation. In the subsequent sections, instead of using the subscript $j$ to represent the star type, we shall note explicitly in parentheses the kind of star and the pulsation mode. For example, the absolute $B$ magnitude for a fundamental mode SX Phe star will be denoted $M_{B}(\mathrm{SX}, \mathrm{F})$. We will also adopt the usual convention of denoting fundamental-mode pulsation periods by $P_{0}$ and firstovertone pulsation periods by $P_{1}$.

\section{3. $P-L$ Relations (in $B$ ) for RR Lyrae stars, Pop. II Cepheids, Anomalous Cepheids and SX Phe Stars}

Fig. 1 shows the apparent $B$ magnitudes versus the pulsation periods for a sample of 710 variable stars (RR Lyraes, P2Cs, ACs and SX Phe variables) in 28 stellar systems. Most of the systems contain two or more of the four kinds of variable stars (e.g., RR Lyrae stars and P2Cs; or, RR Lyraes, ACs and SX Phe's; etc.).

Fig. 2 shows the data with the eight fitted $P$ - $L$ relations for the $\mathrm{F}$ and $\mathrm{H}$ modes for the four different kinds of variable stars. The overall scatter of the observations about all four lines is $\sigma_{B}=0.11 \mathrm{mag}$. In this figure the $B$ magnitudes have been adjusted by taking the Ursa Minor dwarf galaxy, whose RR Lyrae stars have an overall mean magnitude $B=20.16 \mathrm{mag}$, as the zero-point cluster. The $B$ mags for the other systems have been adjusted. It is apparent that the slopes of the $P-L$ relations for the two pulsation modes for the stars of a given kind are approximately parallel. This hypothesis was later tested and found to be a reasonable assumption to within the measuring errors. No metallicity corrections were made, but this did not matter since we were initially interested in the slopes of the $P-L$ relations and not absolute distances.

Although the results of this analysis are encouraging, it does not include the $V$ photometry that is available for all four kinds of stars, or the recently obtained $K$ photometry for approximately $200 \mathrm{RR}$ Lyrae stars. By including the $V$ and $K$ photometry it should be possible to increase the accuracy of the derived distance estimates. In fact, the $K$-band photometry, although limited to a handful of globular clusters, provides some of the most reliable distance information because of the reduced effects of the reddening and metallicity corrections.

For the subsequent analyses we have taken the globular cluster M15 as the zero point cluster, for several reasons: (1) M15 contains over 100 well-studied RR Lyrae stars with varying amounts of $U, B, V$ and $K$ photometry (Sandage, Katem \& Sandage 1981; L90); (2) its color-magnitude diagram is well-defined from the tip of the giant branch to below the main-sequence turnoff (Sandage 1970; Fahlman, Richer \& VandenBerg 1985); (3) the mean metal abundance for M15 is very low and well- 


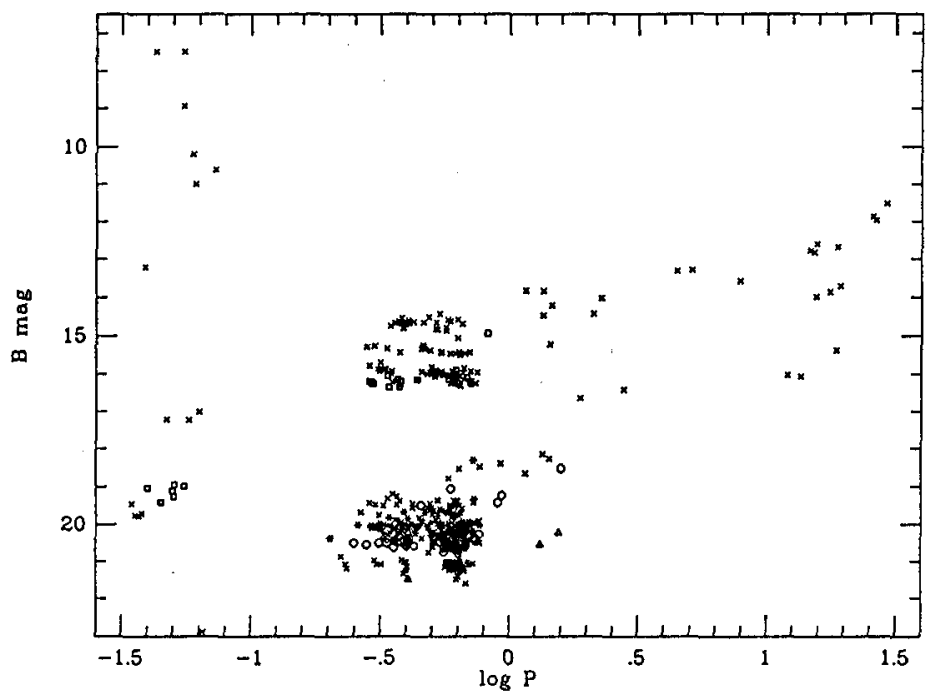

Fig. 1. Period, apparent $B$ magnitude diagram for the program stars. The stars with periods shorter than $\log P=-1$ are SX Phe variables, those with $-0.8 \leq P \leq-0.1$ are mostly RR Lyrae stars (with some possible ACs included), and the remainder are either $\mathrm{ACs}$ or P2Cs.

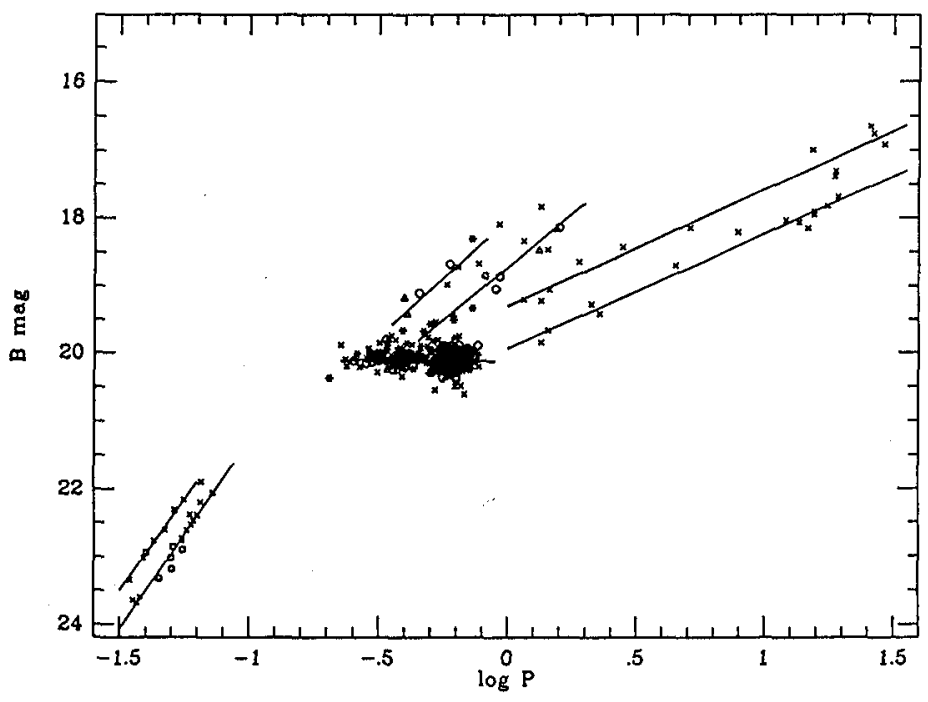

Fig. 2. Period, adjusted $B$ magnitude diagram and $P-L$ relations for the four different kinds of variable stars. The magnitudes have been de-reddened and shifted to the distance of Ursa Minor. Note that the slopes of the $P$ - $L$ relations for the assumed $F$ and $\mathrm{H}$ modes for each kind of variable star are approximately parallel. Subsequent t-tests showed that the assumption of parallel slopes is reasonable and cannot be rejected. 
established at $[\mathrm{Fe} / \mathrm{H}]=-2.15$ (Zinn \& West 1984); furthermore, there is no evidence of star-to-star $[\mathrm{Fe} / \mathrm{H}]$ variations (Sandage \& Katem 1977); (4) the vertical height of the M15 horizontal branch is among the narrowest of any globular cluster (Sandage $1990)$; (5) the reddening is well established at $E(B-V)=0.10 \pm 0.02 ;(6)$ the true distance modulus of M15 is reasonably-well established at $(m-M)_{0}=15.03 \pm 0.05$. The existence of the $U, B, V$ and $K$ photometry of the RR Lyrae stars (L90), and the deep CCD photometry to the level of the main sequence, means that we can relate the variable and non-variable stars; and finally, (7) M15 contains more than 12 double-mode RR Lyrae (RRd) stars whose masses are known (see Petersen 1992), and which permit a tie-in to the theoretical models of the horizontal branch stars.

In the sections that follow we discuss our results for the RR Lyrae stars ( $\$ 4)$, the P2Cs ( $(5)$, the ACs $(\S 6)$ and the SX Phe stars $(\S 7)$. The stars are discussed separately because the photometry available and the issues of interest are different for the four kinds of stars. The magnitude offsets permit us to derive absolute magnitudes to all the stars. Using the relations calibrated by the globular cluster stars it was also possible to derive absolute magnitudes for several field stars (see $§ 7$ ). Our complete results will be presented in detail elsewhere.

\section{4. $P-L-[\mathrm{Fe} / \mathbf{H}]$ Relations in $B, V$ and $K$ for RR Lyrae Stars}

RR Lyrae stars have traditionally been used to derive distance estimates for Pop. II stellar systems. Because they are common in globular clusters, and their $P$ - $L$ relations in $B$ and $V$ are approximately flat, one had, traditionally, only to assume a constant mean luminosity for the RR Lyrae stars in the systems of interest (typically $M_{B}=0.80$ and $M_{V}=0.50$ ), make a reddening correction, and use the difference in the mean magnitudes to obtain a measure of the relative distances. However, it is now clear that this approach is inadequate.

The absolute magnitudes of RR Lyrae stars almost certainly depend on their evolutionary states, which in turn depend on their ages and metal abundances. In a given cluster the mean absolute magnitude of the RR Lyraes appears to correlate with the mean metallicity of the stars, in the sense that the more metal-poor (Oo II) RR Lyraes are more luminous (Sandage 1957, 1990) and more massive (see Simon \& Clement 1993). Sandage (1970) argued that the few RR Lyrae stars in M13 are extra-luminous; and more recently, Lee, Demarque \& Zinn (1990) suggest that the RR Lyrae stars in the most metal-poor systems are in advanced post-ZAHB evolutionary states and are more luminous than the metal-rich (Oo I) RR Lyrae stars (see Lee 1993). Thus, using the 'lower envelope' of the theoretical ZAHB rather than the 'evolutionary mean level' (see Lee 1990, Catelan 1992) would cause the distances for the parent clusters to be underestimated. Furthermore, in addition to systematic effects there are also random effects in that individual stars are found in evolutionary states different from the bulk of the RR Lyrae stars. (The cluster M15 harbors several such stars - Sandage, Katem \& Sandage 1981). Thus, if RR Lyrae stars are to be used as Pop. II distance indicators then these systematic and random effects must be taken into account. Finally, the derived distances must be in accord 
with distance estimates based on main sequence fitting techniques (see Buonanno et al. 1989, and Lee 1993).

We have followed L90, who used the reddening-corrected $K$ mag at $\log P=-0.3$ as their distance indicator, and used the reddening-corrected $B, V$ and $K$ magnitudes at $\log P=-0.3$ as our distance indicators. Thus, our best estimate of the true distance modulus to a particular system is the average of the $B, V$ and $K$ estimates. This assumes that each of the stars in a particular stellar system has the same metal abundance, distance and interstellar extinction (except for the cases noted above), namely, the mean values for the cluster, regardless of the type of variable star. The luminosity, $[\mathrm{Fe} / \mathrm{H}]$ dependence was assumed to be the same for all four kinds of variables, and identical to the slope found by $\mathrm{L} 90$ for the $\mathrm{RR} a b$ stars at $\log P=-0.3$ :

$$
\begin{aligned}
& M_{B}(\mathrm{RR} a b,-0.3)=0.35[\mathrm{Fe} / \mathrm{H}]+1.53, \\
& M_{V}(\mathrm{RR} a b,-0.3)=0.32[\mathrm{Fe} / \mathrm{H}]+1.19, \\
& M_{K}(\mathrm{RR} a b,-0.3)=0.06[\mathrm{Fe} / \mathrm{H}]-0.24 .
\end{aligned}
$$

Table 1 gives the number of RR Lyrae stars (and other kinds of stars) for which $B, V, K$ photometry, and $B-V$ and $V-K$ colors are available (the list of references to be published later). The numbers are summarized separately for the fundamental $(\mathrm{F})$ and first-overtone $(\mathrm{H})$ pulsators.

Table 1

\begin{tabular}{|l|r|r|r|r|r|}
\hline & \multicolumn{5}{|c|}{ Number of Stars } \\
\cline { 2 - 6 } & $B$ & $V$ & $K$ & $B-V$ & $V-K$ \\
\hline TYPE & & & & & \\
\cline { 1 - 6 } Anomalous Cepheids (F) & 13 & 1 & 0 & 1 & 0 \\
\hline Anomalous Cepheids (H) & 15 & 5 & 0 & 5 & 0 \\
\hline Pop. II Cepheids (F) & 19 & 14 & 0 & 14 & 0 \\
\hline Pop. II Cepheids (H) & 22 & 18 & 0 & 18 & 0 \\
\hline RR Lyrae stars (F) & 743 & 414 & 152 & 411 & 101 \\
\hline RR Lyrae stars (H) & 369 & 256 & 51 & 256 & 41 \\
\hline SX Phe stars (F) & 25 & 27 & 0 & 25 & 0 \\
\hline SX Phe stars (H) & 9 & 9 & 0 & 9 & 0 \\
\hline TOTAL & 1215 & 744 & 203 & 739 & 142 \\
\hline
\end{tabular}

Fig. 3 shows the period-'adjusted magnitude' diagrams in $B$ (top panel), $V$ (middle panel) and $K$ (bottom panel) for the 203 RR Lyrae stars that presently have $K$ photometry. The adjusted magnitudes were reddening corrected, normalized to the metallicity of M15, and shifted to the distance of M15. Also shown are the $P-L$ relations computed using the analysis of covariance technique described in $\S 2$. These were 

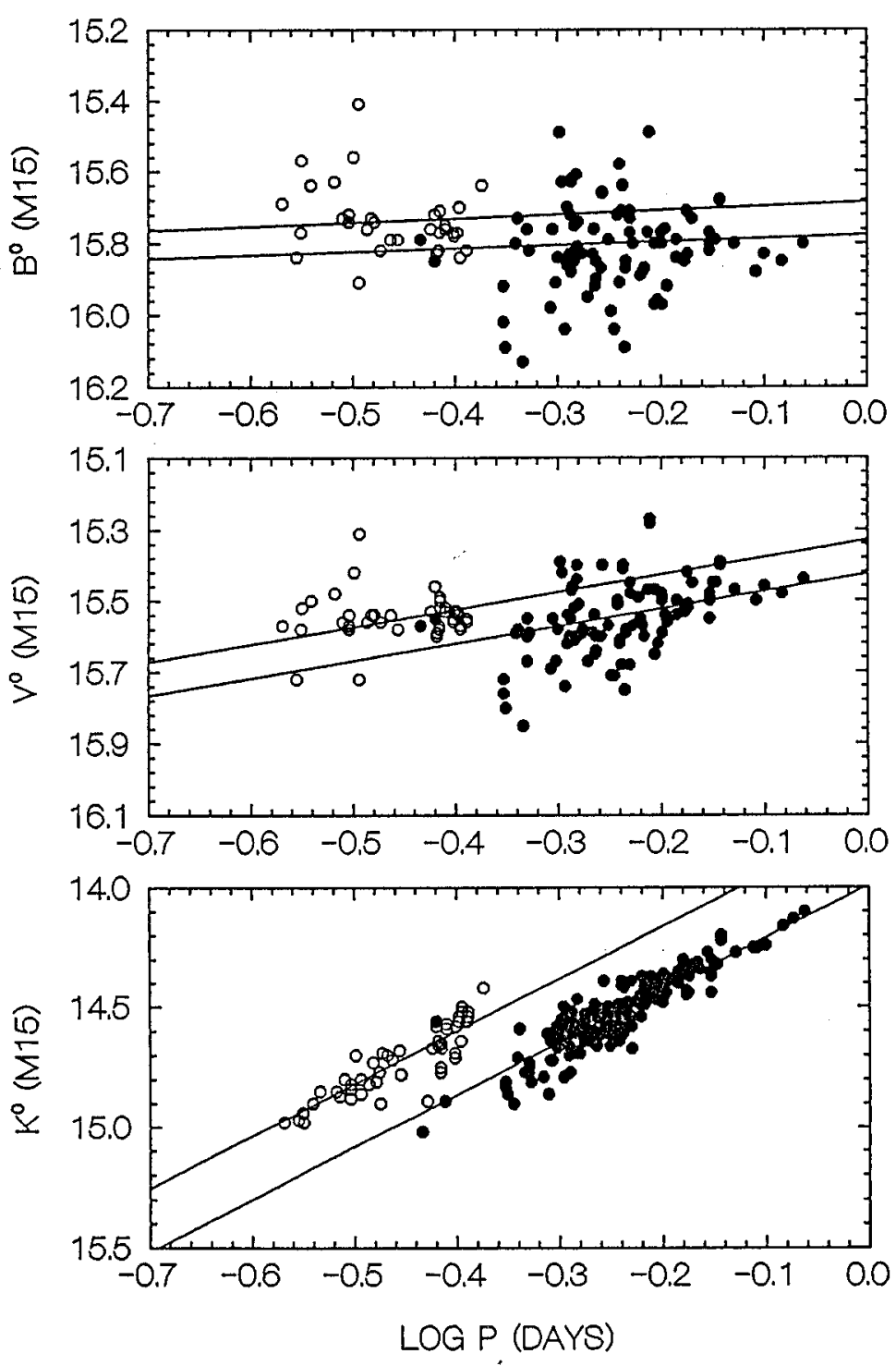

Fig. 3. Period, adjusted magnitude diagrams and $P-L$ relations in $B$ (top panel), $V$ (middle panel), and $K$ (bottom panel) for those globular cluster RR Lyrae stars with $K$ photometry. The magnitudes have been de-reddened, adjusted to the metallicity of M15, and shifted to the distance of M15. The RRab stars are shown as solid circles, and the RRc stars are shown as open circles. The scales are unequal. 
derived by fitting regression lines to the observed pulsation periods and reddeningcorrected, metallicity adjusted magnitudes shifted to the distance of M15.

No evidence was found to suggest that the slopes of the $P-L$ relations in $B, V$ or $K$ for the $F$ and $H$ pulsators are not parallel. The obvious result that for the RR Lyrae stars the $K$ intercepts are different was recovered. Since these tests do not rule out using a single slope for the $P$ - $L$ relation in each passband, we subsequently assumed parallel slopes for the $\mathrm{F}$ and $\mathrm{H}$ stars through all three filters.

Under this assumption, the derived $P-L-[\mathrm{Fe} / \mathrm{H}]$ relations in $B, V$, and $K$ for the RR Lyrae stars are:

$$
\begin{gathered}
M_{B}(\mathrm{RRab})=1.51-0.06( \pm 0.09) \log P_{0}+0.35[\mathrm{Fe} / \mathrm{H}] \\
M_{B}(\mathrm{RRc})=1.44-0.06( \pm 0.09) \log P_{1}+0.35[\mathrm{Fe} / \mathrm{H}] \\
M_{V}(\mathrm{RRab})=1.09-0.44( \pm 0.11) \log P_{0}+0.32[\mathrm{Fe} / \mathrm{H}] \\
M_{V}(\mathrm{RRc})=1.01-0.44( \pm 0.11) \log P_{1}+0.32[\mathrm{Fe} / \mathrm{H}] \\
M_{K}(\mathrm{RRab})=-0.94-2.32( \pm 0.11) \log P_{0}+0.06[\mathrm{Fe} / \mathrm{H}] \\
M_{K}(\mathrm{RRc})=-1.23-2.32( \pm 0.11) \log P_{1}+0.06[\mathrm{Fe} / \mathrm{H}]
\end{gathered}
$$

Of interest here is the statistically significant increase in the slopes of the $P$ - $L$ relations in going from the blue to the infrared. The existence of a non-zero slope for the $V$ photometry also means that one should be cautious when dealing with a small number of RR Lyrae stars in using a simple mean $V$ magnitude in estimating distances. Notice that, since much of the same data were analyzed and the $M_{K}-[\mathrm{Fe} / \mathrm{H}]$ relation of $\mathrm{L} 90$ was assumed, the $M_{K}(\mathrm{RRab})$ relation is in good agreement with equation (9) of L90 (see also Liu \& Janes 1993, and equation 6 of Longmore 1993).

\section{Pop. II Cepheids: Fundamental and First-Overtone Pulsators?}

In Arp's (1955) analysis of the P-L relations for globular cluster Cepheids (in $m_{p g}$ and $m_{p v}$ ) he concluded that these stars pulsate in the fundamental and first-overtone modes (and possibly in higher pulsation modes), and that the stars of each mode follow separate $P-L$ relations offset from and parallel to each other. Since the early 1970's this hypothesis has been largely ignored in favor of the assumption that all $\mathrm{P} 2 \mathrm{Cs}$ are $\mathrm{F}$ pulsators obeying a single $P-L$ relation. For example, based on 17 stars in the globular clusters $\omega$ Cen, M2, M13 and M14, Demers \& Harris (1974) obtained the single line $M_{V}=-1.59 \log P-0.08$, with a residual scatter of $\pm 0.21 \mathrm{mag}$. More recently, Harris (1985) assumed the Demers \& Harris relation for stars with periods less than 12.5 days, and adopted a steeper relation $M_{V}=-3.6 \log P+2.2$ for longer period stars. In both papers the scatter about the $P-L$ relation is as much as 0.3 mag at a given period.

Fig. 4 shows the period, adjusted magnitude diagrams in $V$ (top panel) and in $B$ (middle panel) for the P2Cs. Also shown are the derived $P-L$ relations. For a given filter common slopes were assumed in the calculation for the $\mathrm{F}$ and $\mathrm{H}$ modes, after no evidence was found that the slopes of the $P-L$ relations are not parallel. 

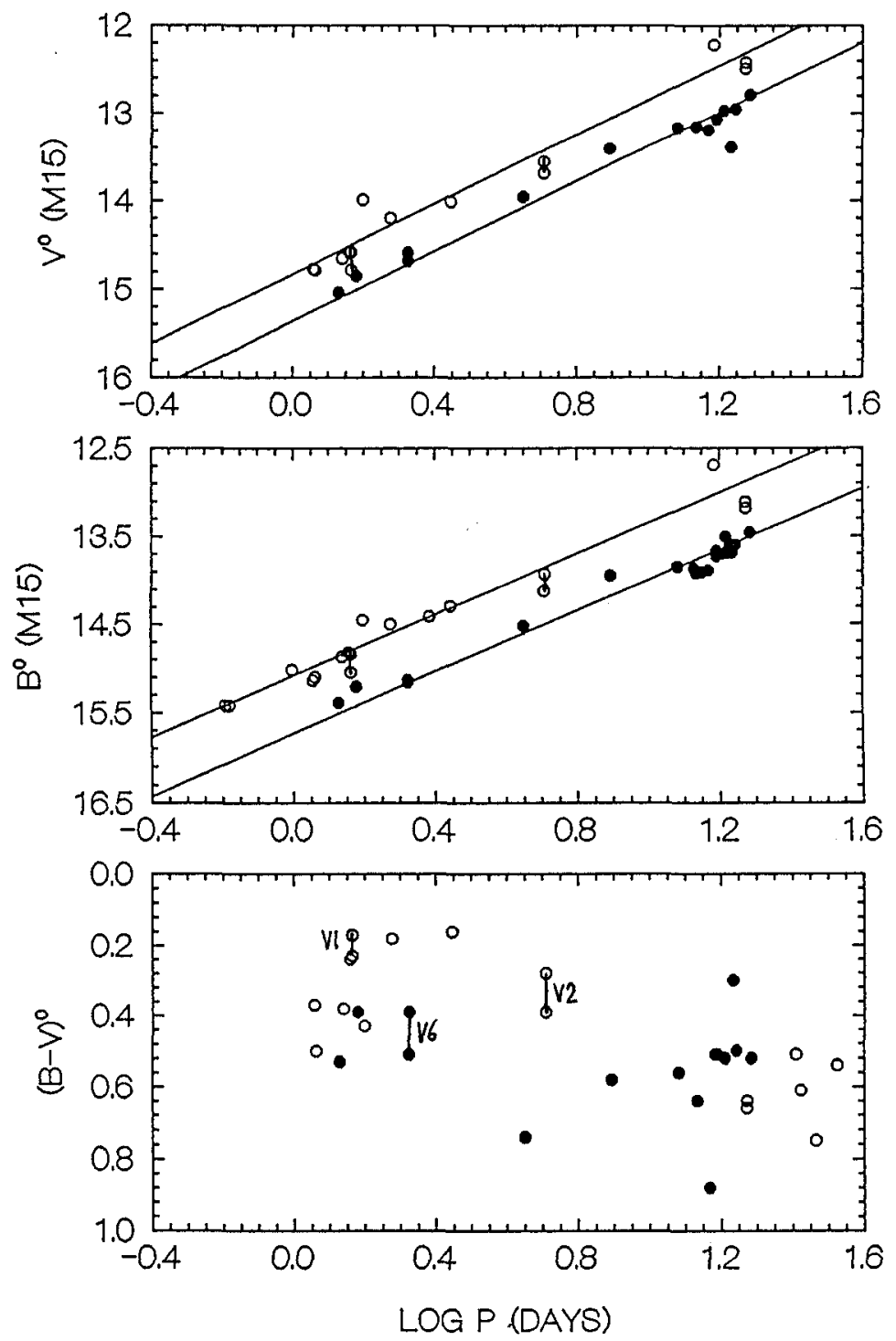

Fig. 4. Period, adjusted magnitude diagrams and $P-L$ relations in $V$ (top panel) and in $B$ (middle panel) for the globular cluster Cepheids. The magnitudes have been de-reddened and metallicity corrected and shifted to the distance of M15. At a given period the assumed $F$ pulsators (solid circles) are seen to be, on average, less luminous than the assumed $H$ pulsators (open circles). The P-C diagram (bottom panel) shows that the short-period Pop. II Cepheids (the BL Her stars) are systematically bluer than the long-period Cepheids (W Vir stars), and that at a given pulsation period the first-overtone stars are systematically bluer than the fundamental-mode stars. 
The lines have slopes of $-1.72(B)$ and $-1.96(V)$. The derived $B$ slopes were found to lie midway between the corresponding slopes shown in Fig. 2. The new slope is steeper than the Demers-Harris slope, in accord with Harris' modification for the longer period stars; however, in our model there is no need for a change in slope at mid-periods.

The bottom panel of Fig. 4 shows the period-color $(P-C)$ diagram for the P2Cs. Clearly the longer-period (W Vir) Cepheids are redder on average than the shorterperiod (BL Her) stars, a result seen previously in Fig. 1 of Harris (1985). A linear $P-C$ relation through all the points was derived by Harris: $(B-V)=0.275+0.206 \log P$. In addition to supporting this result, the bottom panel of Fig. 4 suggests that the firstovertone BL Her stars are bluer than the fundamental-mode BL Her stars, paralleling the situation for the slightly lower-luminosity $R R$ Lyrae variables. At longer periods and higher luminosities the discrimination between $F$ and $H$ stars is reduced and there is no obvious color dependence.

Fig. 5 shows the period, absolute magnitude diagrams in $V$ (top panel) and in $B$ (bottom panel) for the P2Cs. The $P-L-[\mathrm{Fe} / \mathrm{H}]$ families of lines (not plotted) are given by:

$$
\begin{aligned}
& M_{B}(\mathrm{P} 2 \mathrm{C}, \mathrm{F})=1.42-1.72( \pm 0.05) \log P_{0}+0.35[\mathrm{Fe} / \mathrm{H}] \\
& M_{B}(\mathrm{P} 2 \mathrm{C}, \mathrm{H})=0.77-1.72( \pm 0.05) \log P_{1}+0.35[\mathrm{Fe} / \mathrm{H}] \\
& M_{V}(\mathrm{P} 2 \mathrm{C}, \mathrm{F})=0.97-1.96( \pm 0.05) \log P_{0}+0.32[\mathrm{Fe} / \mathrm{H}] \\
& M_{V}(\mathrm{P} 2 \mathrm{C}, \mathrm{H})=0.44-1.96( \pm 0.05) \log P_{1}+0.32[\mathrm{Fe} / \mathrm{H}]
\end{aligned}
$$

Because the $[\mathrm{Fe} / \mathrm{H}]$ range of the Cepheids is only from -1.39 (for M14) to -2.15 (for M15) the scatter at a given period is not much larger than that seen in Fig. 4.

The success with which the $P-L$ relations in Figs. 2 and 4 fit the P2C data suggest that Arp's assumption of two or more distinct $P-L$ relations for $P 2 C s$, with the brighter stars at a given period being first-overtone pulsators and the fainter stars being fundamental-mode pulsators, is correct. If $\mathrm{P} 2 \mathrm{Cs}$ do pulsate in the $\mathrm{F}$ and $\mathrm{H}$ modes there would be an appealing symmetry with the well-known $a b$ - and $c$-type RR Lyrae stars, with the ACs and SMC Cepheids that almost certainly follow two parallel lines (see $\S 6$ ), with the SX Phe stars that obey two $P-L$ relations (see $\S 7$ ), as well as with the Pop.I Cepheids that are now being discovered in young Magellanic Cloud clusters (see Welch et al. 1993).

Arp's hypothesis also explains a number of outstanding puzzles, including the problem of the Cepheids V1, V2 and V6 in M13 (labelled in the bottom panel of Fig.4 - the observations of Pike \& Meston 1977, and of Demers \& Harris 1974, were treated separately and both sets of data have been plotted). These stars were studied extensively by Böhm-Vitense et al. (1974). They noted that, while V6 has a reddish color and an effective temperature consistent with pulsation in the $\mathrm{F}$ mode, the very blue colors and early spectral types (A2 - Joy 1949) of V1 and V2 are anomalously bluer than the theoretical fundamental-mode blue edges for various assumed helium abundances. Böhm-Vitense et al. argue that if the relatively high temperatures of $\mathrm{V} 1$ and V2 are to be believed, then both stars must have very low masses ( 0.3-0.4 
solar masses) and relatively high envelope helium abundances ( $\sim 0.6-0.7)$. Our fits suggest that V1 and V2 lie on the brighter of the two P2C P-L lines plotted in Fig. 4, and that V6 lies on the lower of the two lines, and thus V1 and V2 are $\mathrm{H}$ pulsators while V6 is an F pulsator. This being the case there is no longer the need to suggest unusual mases or abundances.

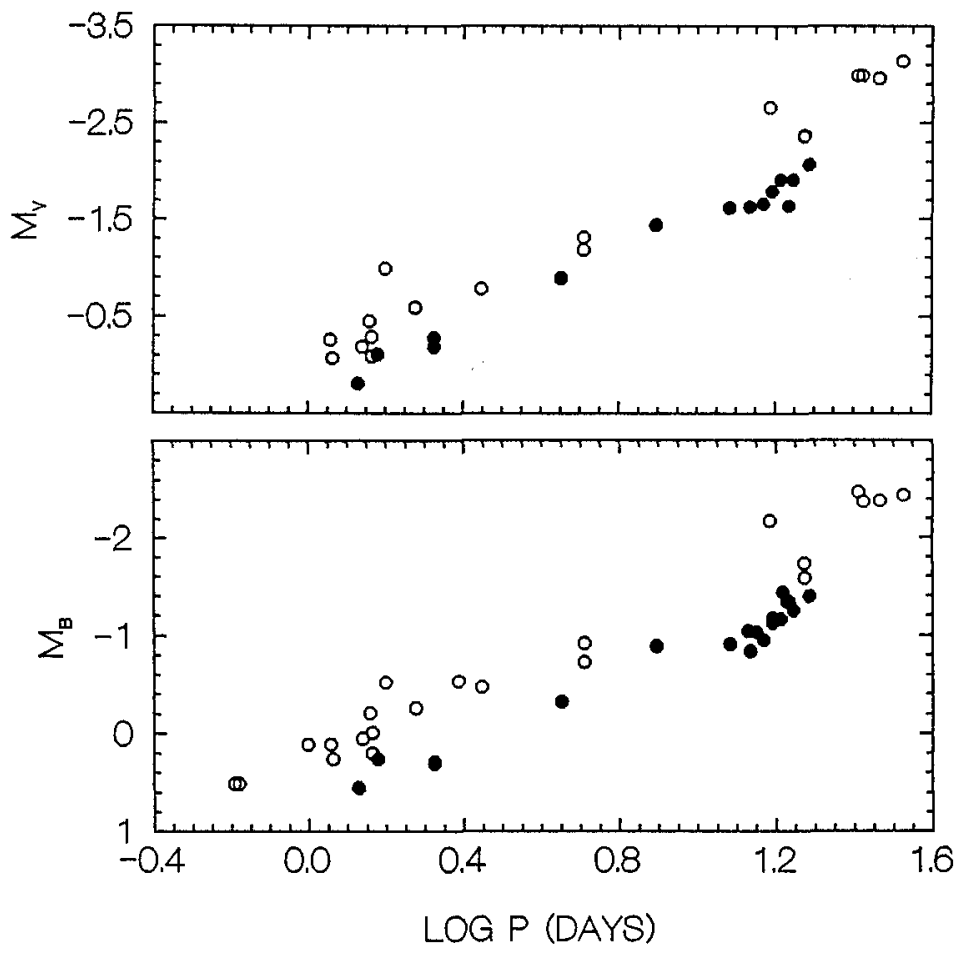

Fig. 5. Period, absolute magnitude diagrams for the globular cluster Cepheids.

If the assignment of pulsation modes based on the $P-L$ diagram is valid, then one might expect there to be some evidence of the different pulsation modes in the light amplitudes and shapes of the light curves. This is certainly the case for the RR Lyrae stars, where the period-amplitude $(P-A)$ diagram shows well-defined differences and correlations, with the $a b$-type RR Lyrae stars tending to have larger amplitudes than the $c$-type stars (except at small amplitudes where the two amplitude distributions overlap). Furthermore, for a given metallicity, the amplitudes of the $a b$-type stars exhibit a linear trend with $\log P$, in the sense that the shorter- $P$ stars have larger amplitudes than the longer- $P$ stars. The lower metallicity stars exhibit the same overall amplitude trends as do higher metallicity stars but with the $\log P$-A relation shifted toward longer periods at a given amplitude (temperature).

A more quantitative use of light curve morphology for mode assignment might be possible if results of Fourier decomposition analyses were used (see Simon \& Lee 
1981, Antonello et al. 1986, and Petersen 1993). Unfortunately, most P2Cs lack welldefined light curves based on modern observations and hence such analyses are not presently possible. Alternatively, one could use period-amplitude diagrams to sort $\mathrm{F}$ and $\mathrm{H}$ stars, and the periods of double-mode P2Cs to establish better the separation of the two parallel lines.

Until such analyses have been carried out, we propose (based on the $P-L, P-C$ and $P-A$ diagrams) the following mode assignments: N2419-V18(H); $\omega$ Cen-V1(H), $\mathrm{V} 29(\mathrm{~F}), \mathrm{V} 43(\mathrm{H}), \mathrm{V} 48(\mathrm{~F}), \mathrm{V} 60(\mathrm{H}), \mathrm{V} 61(\mathrm{~F}), \mathrm{V} 92(\mathrm{~F}) ; \mathrm{M} 3-\mathrm{V} 154(\mathrm{H}) ; \mathrm{M} 5-\mathrm{V} 42(\mathrm{H}), \mathrm{V} 84(\mathrm{H})$; M80-V1(F); M10-V1(F), V2(H), V3(F); M13-V1(H), V2(H), V6(F); M19-V1(F), V2(F), $\mathrm{V} 3(\mathrm{~F}), \mathrm{V} 4(\mathrm{H}) ; \mathrm{M} 14-\mathrm{V} 1(\mathrm{~F}), \mathrm{V} 2(\mathrm{H}), \mathrm{V} 7(\mathrm{~F}), \mathrm{V} 17(\mathrm{~F}), \mathrm{V} 76(\mathrm{H}) ; \mathrm{M} 28-\mathrm{V} 4(\mathrm{~F}), \mathrm{V} 5(\mathrm{H})$, $\mathrm{V} 9(\mathrm{H}), \mathrm{V} 22(\mathrm{H}) ; \mathrm{N} 6752-\mathrm{V} 1(\mathrm{H}) ; \mathrm{M} 15-\mathrm{V} 1(\mathrm{H}), \mathrm{V} 72(\mathrm{H}), \mathrm{V} 86(\mathrm{~F}) ;$ and M2-V1(F), V5(F), $\mathrm{V} 6(\mathrm{~F})$.

\section{6. $P-L-[\mathrm{Fe} / \mathrm{H}]$ Relations in $B$ and $V$ for Anomalous Cepheids}

Anomalous Cepheids have been extensively reviewed by Zinn (1985a), DaCosta (1988) and Nemec (1989). Most of the 50 ACs that are known are located in seven nearby dwarf galaxies (see Table IX of Nemec, Wehlau \& Oliveira 1988, hereafter NWO). In addition, at least one such star is known in the galactic globular cluster NGC 5466 (Zinn \& King 1982), and several ACs have recently been identified in the Magellanic Cloud globular cluster NGC 1786 (Walker \& Mack 1988). At a given luminosity ACs tend to have shorter periods than the Cepheids found in globular clusters, which can be explained if the stars are more massive than the P2Cs. The leading explanation for the ACs is that they are coalesced binary systems above the horizontal branch in the Cepheid instability strip and that they are possibly related to the blue stragglers found in the same systems.

NWO showed that ACs obey two distinct linear $P-L$ relations, one for the F mode pulsators and one for the $\mathrm{H}$ pulsators. They determined the relations by shifting the mean magnitudes of the RR Lyrae stars in the individual clusters to the mean apparent magnitude for the Ursa Minor RR Lyrae stars, at $B=20.16 \mathrm{mag}$. The absolute magnitudes were then found to be in the range $-1.5 \leq M_{B} \leq 0.4$ by assuming that the mean absolute magnitude for the UMi RR Lyrae stars is $M_{B}=0.80$. The resulting $P-L$ relations seemed to diverge.

Fig. 6 shows our period, adjusted magnitude diagrams in $B$ (middle panel) and in $V$ (upper panel), and the new $P-L$ solutions. The top panel shows that in $V$ only the $P$ - $L$ relation for the first-overtone ACs is available. In contrast to the result of NWO, no evidence for a lack of parallelism for the $\mathrm{F}$ and $\mathrm{H}$ pulsators ( $c f$. Fig.6, and Fig.13 of NWO) was found for the slopes of the $P$ - $L$ relations in either $B$ or $V$. This change was brought about in part by the existence of the new Goldsmith (1993) Sculptor RR Lyrae star photometry that constrains the luminosities of the Sculptor $\mathrm{ACs}$ and has resulted in revised mode classifications for V25 and V26 from $\mathrm{F}$ to $\mathrm{H}$.

Fig. 7 shows the period, absolute magnitude diagrams in $V$ (top panel) and in $B$ (bottom panel) for the ACs. The large scatter at a given period is due to the large range in metallicity of the stars, from approximately -0.60 for the SMC field stars, 

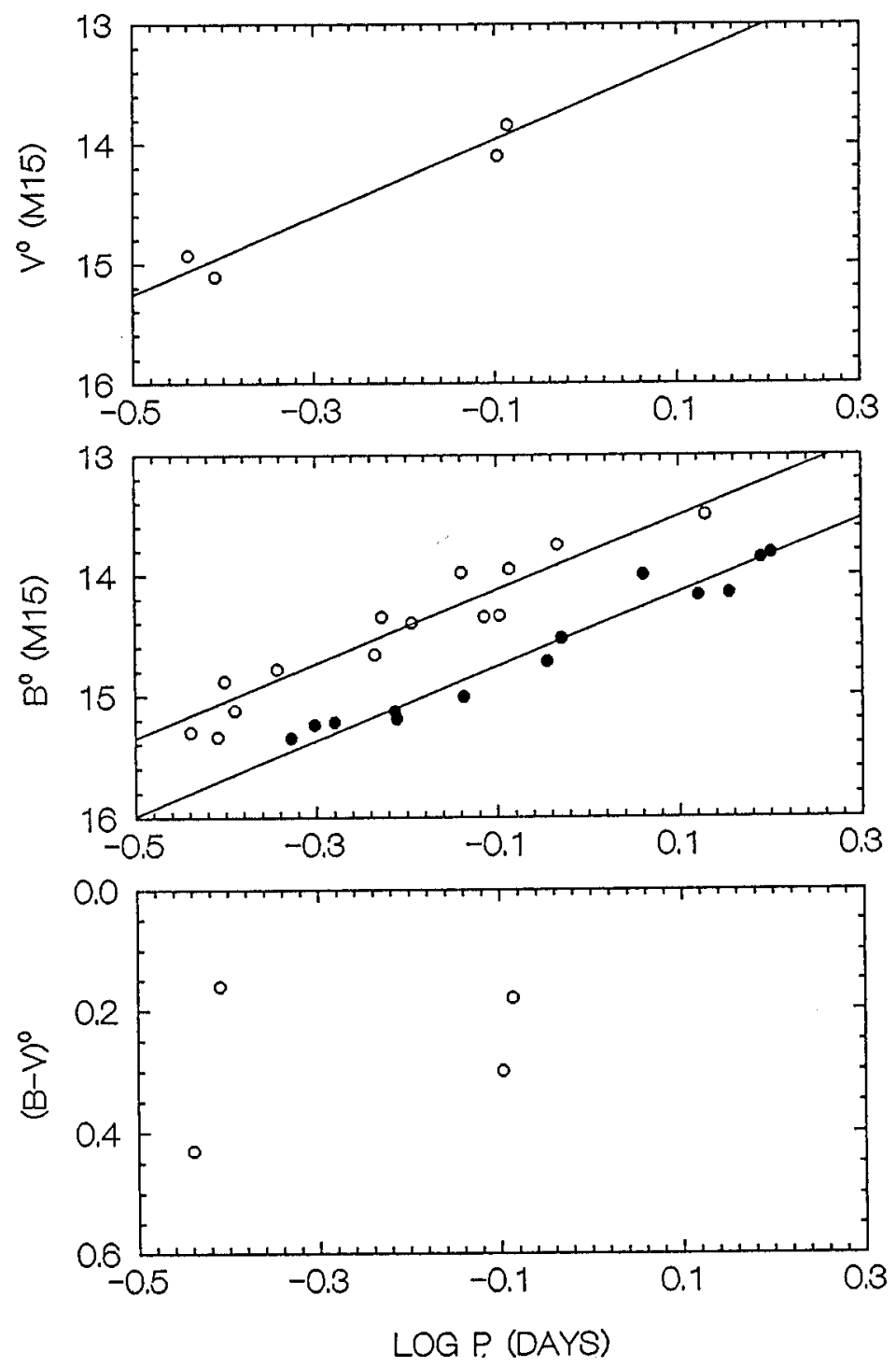

Fig. 6. Period, adjusted magnitude diagrams in $V$ (top panel) and in $B$ (middle panel) for the anomalous Cepheids. The assumed $F$ and $H$ pulsators are show as solid circles and open circles, respectively. The photometry has been reddening corrected, adjusted to the metallicity of M15, and shifted to the distance of M15. Parallel slopes were assumed for the two modes. Clearly more photometry is needed before anything substantive can be said about possible period-color (bottom panel) relations. 
to -2.02 for the Ursa Minor ACs. The derived $P-L-[\mathrm{Fe} / \mathrm{H}]$ relations are as follows:

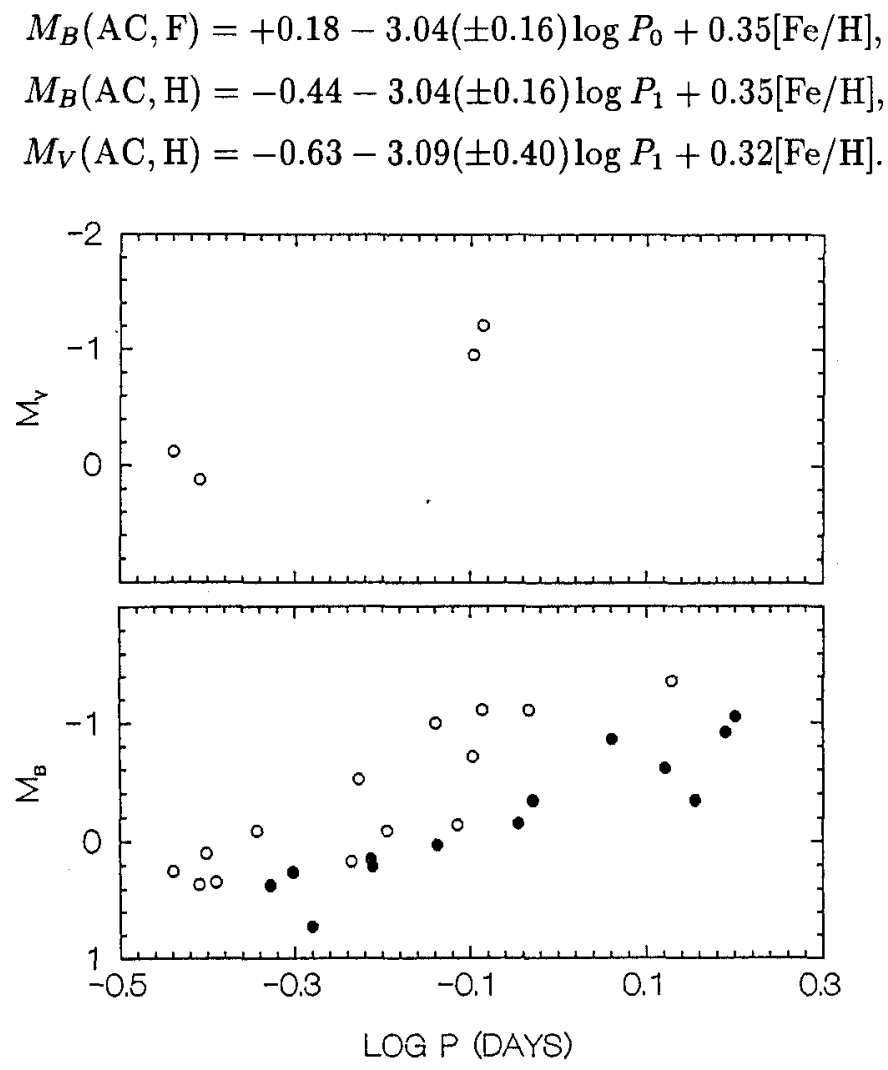

Fig. 7. Period, absolute magnitude diagrams for the anomalous Cepheids. The large scatter at a given period seen in the lower panel is due mainly to the different metallicities of the program stars.

It has long been thought that the Cepheids in the Small Magellanic Cloud (SMC) may be related to the anomalous Cepheids found in the dwarf galaxies. Recently, Smith et al. (1992, hereafter SSBG) published the results of an extensive investigation of the variable stars in a NE region of the SMC. They present photographic $B$ photometry and derive the $P-L$ characteristics for a large number of short-period Cepheids. The clean division of the SMC Cepheids into two groups with nearly parallel $\log P-M_{B}$ relations, just as the ACs studied here, is of considerable interest. The $P-L$ relations derived by SSBG for the F and $\mathrm{H}$ pulsators in the SMC were found to be:

$$
\begin{aligned}
& B(\mathrm{SMC}, \mathrm{F})=-2.71( \pm 0.09) \log P_{0}+18.29( \pm 0.04), \\
& B(\mathrm{SMC}, \mathrm{H})=-2.98( \pm 0.17) \log P_{1}+17.61( \pm 0.04) .
\end{aligned}
$$

Since the separation of the two lines at $\log P=-0.1$ (the center of the range in $\log P$ for the ACs) is $0.65 \mathrm{mag}$, compared with the $0.66 \mathrm{mag}$ for the ACs found here, 
and since the slopes derived by SSBG, -2.71 and -2.98 , are very close to the slope $-3.04 \pm 0.16$ derived for the ACs, our results for the ACs appear to be consistent with those of SSBG for the SMC Cepheids. At the very least, the identification of the two modes with $F$ and $H$ pulsation seems secure. To settle the matter it would be useful to have metal abundances for the SMC Cepheids and for the ACs to remove the confounding effects of the metallicities on the distance determinations so that a direct comparison can be made.

\section{7. $P-L-[\mathrm{Fe} / \mathrm{H}]$ relations in $B$ and $V$ for $\mathbf{S X}$ Phe Variables}

Over 30 SX Phe stars are now known in several globular clusters: three in $\omega$ Cen (Jörgensen \& Hansen 1984), six in NGC 5466 (Mateo et al. 1988; 1993, in preparation), five in NGC 5053 (Nemec 1989; Nemec et al. 1993, in preparation), one in M71 (Hodder et al. 1992), one (candidate) star in M3 (DaCosta 1988), and eight which were recently discovered in NGC 4372 (Kaluzny \& Krzeminski 1992 preprint). In addition five field SX Phe stars (BL Cam, DY Peg, SX Phe, CY Aqr and KZ Hya) are also known (see Nemec \& Mateo 1990). Fig. 7 of Nemec (1989) shows an early $\log P-M_{V}$ diagram that included most of these stars, with the $P-L$ relations for the suspected $\mathrm{F}$ and $\mathrm{H}$ stars drawn through the $V$ data available at that time. Also shown was a period-amplitude $\left(\log P-A_{V}\right)$ diagram showing that the visual amplitudes of SX Phe stars tend to increase with lengthening pulsation period.

In Fig. 8 period, adjusted magnitude diagrams for the SX Phe stars are plotted in $V$ (top panel) and in $B$ (middle panel), and in the bottom panel we plot the period-color diagram. To obtain estimates of the $P-L$ relations, two cases, both of which assumed parallel slopes, were analyzed. The upper diagrams show the least squares lines derived without the RR Lyrae stars in the globular clusters NGC 5053, NGC 5466 and $\omega$ Cen. The reason for their exclusion was that the present photometry of the RR Lyrae stars in these systems is very uncertain. In all three panels of Fig. 8 the well-known double-mode star SX Phe has been plotted twice, once for the firstovertone component, and once for the fundamental-mode component, with the two points connected by a line. It is encouraging that its accurately-known period ratio, $P_{1} / P_{0}=0.7782$, is very close in value to the horizontal separations of the $\mathrm{F}$ and $\mathrm{H}$ lines (i.e., period ratios, $\left.P_{1} / P_{0}\right)$ of $0.81(B)$ and $0.83(V)$. The differences between the observed and predicted period ratios can be attributed to measurement errors and the small sample sizes (particularly for the $\mathrm{H}$ pulsators). Other field SX Phe stars are also plotted in the diagrams, at their fitted positions on the lines.

The derived $P-L-[\mathrm{Fe} / \mathrm{H}]$ equations for the case where the RR Lyrae stars in NGC 5053, NGC 5466 and $\omega$ Cen were excluded, are given by

$$
\begin{aligned}
& M_{B}(\mathrm{SX}, \mathrm{F})=-3.99-5.44( \pm 0.75) \log P_{0}+0.35[\mathrm{Fe} / \mathrm{H}] \\
& M_{B}(\mathrm{SX}, \mathrm{H})=-4.53-5.44( \pm 0.75) \log P_{1}+0.35[\mathrm{Fe} / \mathrm{H}] \\
& M_{V}(\mathrm{SX}, \mathrm{F})=-4.10-5.42( \pm 0.70) \log P_{0}+0.32[\mathrm{Fe} / \mathrm{H}] \\
& M_{V}(\mathrm{SX}, \mathrm{H})=-4.60-5.42( \pm 0.70) \log P_{1}+0.32[\mathrm{Fe} / \mathrm{H}]
\end{aligned}
$$



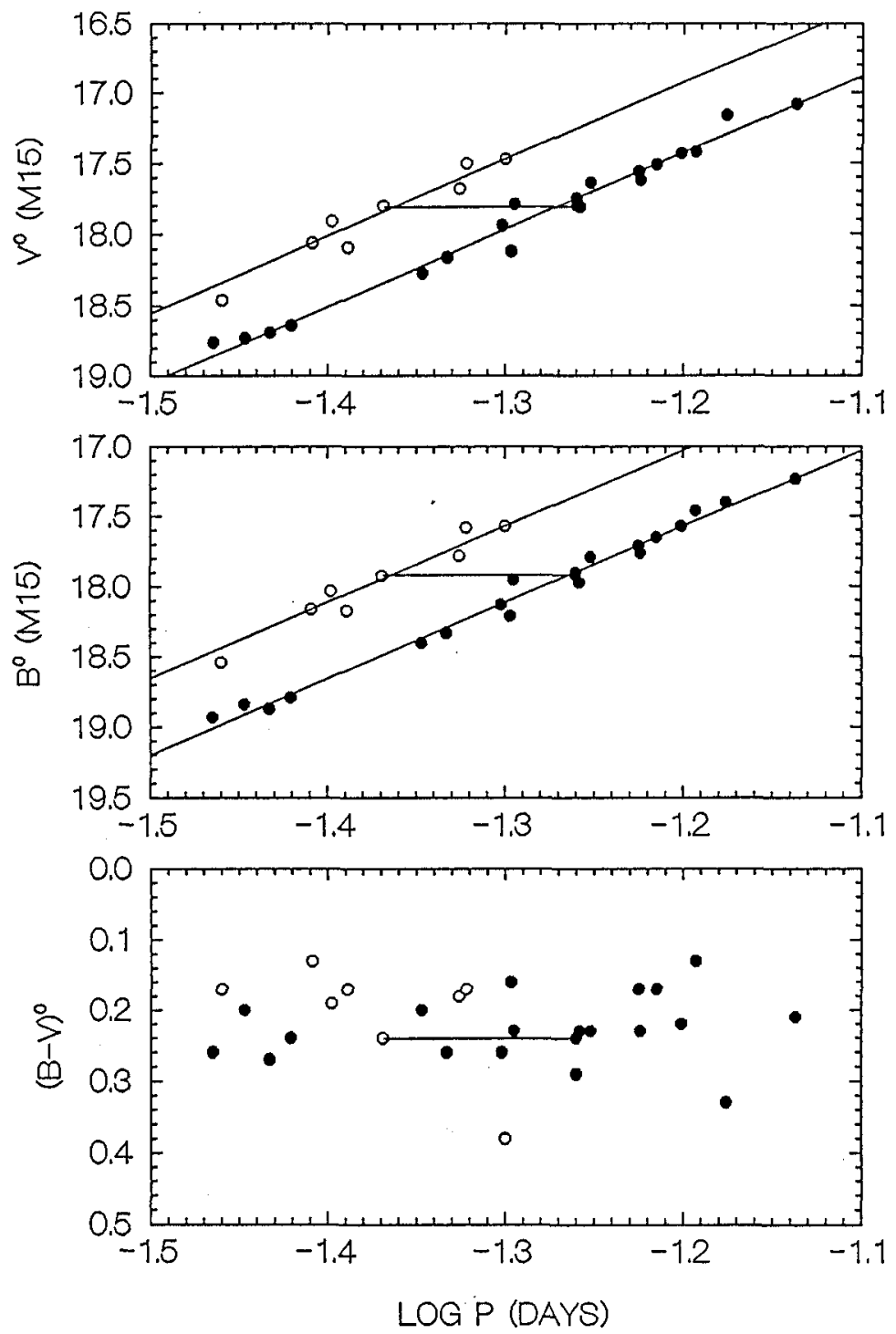

Fig. 8. Period, adjusted magnitude diagrams and $P-L$ relations in $V$ (top panel) and in $B$ (middle panel) for the SX Phe stars. The assumed fundamental-mode (F) pulsators are plotted as solid circles, and the assumed first-overtone $(H)$ pulsators are represented by open circles. Both periods for SX Phe are shown, connected by horizontal lines. The photometry has been reddening corrected, adjusted for metallicity differences, and shifted to the distance of the globular cluster M15. In computing the $P-L$ relations parallel slopes were assumed for the two modes. The $P$-C diagram (bottom panel) shows that at a given period the $\mathrm{H}$ pulsators tend to be bluer than the $\mathrm{F}$ pulsators (an exception being $\mathrm{H} 1$ in M71 at $\log P=-1.3)$. 
These are shown in Fig. 9, where we have plotted the corresponding period, absolute magnitude diagrams in $V$ (top panel) and in $B$ (bottom panel).
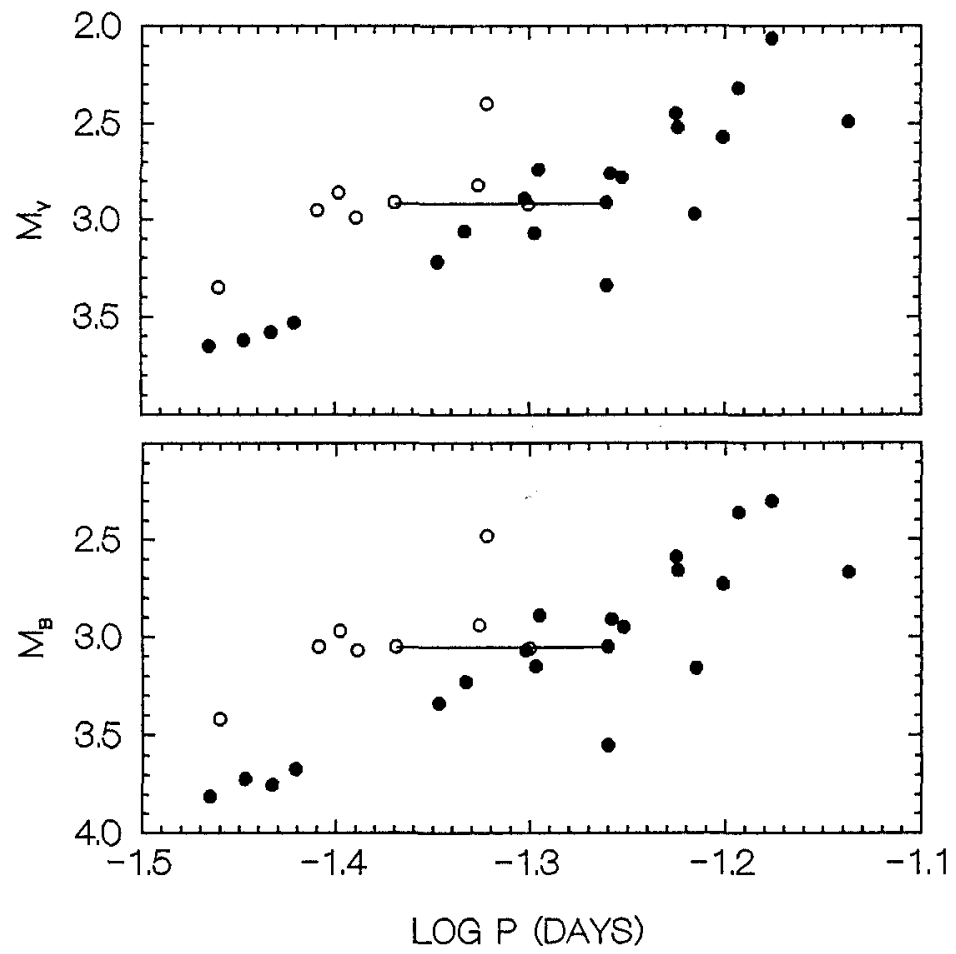

Fig. 9. Period, absolute magnitude diagrams for the SX Phe stars. The large scatter at a given period seen at the longer periods is mainly due to the metal richness of the SX Phe stars in the field compared with those in the globular clusters.

When the RR Lyrae stars in $\omega$ Cen, NGC 5466 and NGC 5053 were included in the analysis the family of $P-L-[\mathrm{Fe} / \mathrm{H}]$ lines was found to be:

$$
\begin{aligned}
& M_{B}(\mathrm{SX}, \mathrm{F})=-1.85-4.44( \pm 0.74) \log P_{0}+0.35[\mathrm{Fe} / \mathrm{H}], \\
& M_{B}(\mathrm{SX}, \mathrm{H})=-2.27-4.44( \pm 0.74) \log P_{1}+0.35[\mathrm{Fe} / \mathrm{H}], \\
& M_{V}(\mathrm{SX}, \mathrm{F})=-1.71-4.19( \pm 0.70) \log P_{0}+0.32[\mathrm{Fe} / \mathrm{H}] \\
& M_{V}(\mathrm{SX}, \mathrm{H})=-2.05-4.19( \pm 0.70) \log P_{1}+0.32[\mathrm{Fe} / \mathrm{H}]
\end{aligned}
$$

The slopes in this case agree at the 0.05 level of significance with the preceding estimates, although the scatter about the $P$ - $L$ lines is somewhat greater as expected. If the photometry of the NGC 5053, NGC 5466 and $\omega$ Cen RR Lyrae stars were more reliable this would be the preferred solution. However, with the available data we can only conclude that the slope is approximately $-5.0 \pm 0.5$. The results of the photometry projects underway by H.Harris et al. for the RR Lyrae stars in NGC 5466, and by Corwin et al. for the RR Lyrae stars in NGC 5053 are eagerly awaited. 
Fig. 8 and Fig. 9 also show the field SX Phe stars BL Cam, KZ Hya and DY Peg (all are on the $P-L$ lines in the upper two panels of Fig. 8). Assuming that the metallicities, reddenings, and pulsation modes for these stars are as given by Nemec \& Mateo (1990), it is possible to compute their absolute magnitudes using the above equations. For example, for SX Phe with $[\mathrm{Fe} / \mathrm{H}]=-1.70$ and $P_{0}=0.055 \mathrm{day}$, the second set of equations gives $M_{B}=2.7 \pm 0.6$ and $M_{V}=2.6 \pm 0.5$. It will be of considerable interest when the zero-points of the $P-L-[\mathrm{Fe} / \mathrm{H}]$ relations are set using the accurate parallax for SX Phe obtained by HIPPARCOS.

\section{Summary}

Period-luminosity-metallicity relations in $B, V$ and $K$ have been derived for RR Lyrae stars, anomalous Cepheids, Pop. II Cepheids and SX Phe stars in globular clusters. For all four kinds of stars the assumption that the stars pulsate either in the fundamental or first-overtone modes appears to be reasonable (obvious exceptions are the double-mode stars and the Blazhko RR Lyrae stars). Statistical tests show that the hypohesis of equal slopes for the $\mathrm{F}$ and $\mathrm{H} P-L$ relations in each filter cannot be rejected. For the P2Cs it was found that the majority of the shorter-period Cepheids pulsate in the first-overtone mode, a result anticipated by Arp in 1955 but largely ignored since then. The displacements of the parallel $P-L-[\mathrm{Fe} / \mathrm{H}]$ lines for the RR Lyrae and SX Phe stars are in agreement with the well-known period ratios for double-mode RR Lyrae and SX Phe stars (0.746 and 0.778, respectively). This agreement lends support to the method of mode assignment based on the $P$ - $L$ diagram. It was also found that the SMC Cepheids studied by Smith et al. (1992) are similar to the anomalous Cepheids, with comparable slopes and separation for the $P$ - $L$ relations; however, we cannot be sure that they are the same kind of star. Finally, we note that the period-amplitude $(P-A)$ relations for the SX Phe and $\mathrm{P} 2 \mathrm{Cs}$ are somewhat unusual because the $\mathrm{H}$ stars tend to have, at a given period, larger amplitudes than the $\mathrm{F}$ pulsators. Complete details of our analyses will be presented elsewhere.

In the future, $P-L-[\mathrm{Fe} / \mathrm{H}]$ relations should provide more accurate absolute magnitudes for the individual stars and estimates of relative distances for the parent stellar systems. It would be highly desirable to identify double-mode $\mathrm{P} 2 \mathrm{Cs}$ and $\mathrm{ACs}$, since these will serve to confirm the separations between the $P-L-[\mathrm{Fe} / \mathrm{H}]$ lines for the $\mathrm{ACs}$ and P2Cs. It will also be useful to obtain improved photometry (and more $K$ band photometry) that will be useful for refining the results presented here, as well as for Fourier decomposition purposes. It would also be valuable to have a theoretical explanation for the observed period-amplitude relations.

We thank Amanda F. Linnell Nemec for useful discussions and assistance with the SAS package and ANCOVA methods, and acknowledge Erika Böhm-Vitense, Robert Buchler, Guillermo Gonzalez, Hugh Harris, Young-Wook Lee, Pawel Moskalik, Allan Sandage, Norman Simon and George Wallerstein for useful discussions and preprints. J.M.N. gratefully acknowledges an NSERC operating grant and an adjunct professorship at York University. 


\section{References:}

Antonello, E., Broglia, P., Conconi, P. \& Mantegazza, L. 1986, A\&A, 169, 122.

Arp, H.C. 1955, AJ, 60, 1.

Böhm-Vitense, E. 1988, ApJ, 324, L27.

Böhm-Vitense, E., Szkody, P., Wallerstein, G. \& Iben, I. 1974, ApJ, 194, 125.

Buonanno, R., Corsi, C.E., Fusi Pecci, F. 1989, A\&A, 216, 80.

Buonanno, R., Cacciari, C., Corsi, C.E., Fusi Pecci, F. 1990, A\&A, preprint.

Cacciari, C. 1984, AJ, 89, 231.

Catelan, M. 1993, these proceedings, p.271.

Da Costa, G. 1988, In The Harlow Shapley Symposium on Globular Cluster Systems in Galaxies, IA U Symp 126, ed. J.Grindlay \& A.G.D.Philip, Dordrecht:Reidel, p.217.

Demers, S. \& Harris, W.E. 1974, AJ, 79, 627.

Fahlman, G.G., Richer, H.B. and VandenBerg, D.A. 1985, ApJ, 58, 25.

Fernie, D. 1992, AJ, 103, 1647.

Goldsmith, C. 1993, these proceedings, p.347

Harris, H.C. 1985, In Cepheids: Theory and Observations ed. B.F.Madore, Cambridge University Press, p.232.

Hodder, P.J.C., Nemec, J.M., Richer, H.B. \& Fahlman, G.G. 1992, AJ, 103, 460.

Jörgensen, H. \& Hansen, L. 1984, A\&A, 133, 165.

Joy, A. H. 1949, ApJ, 110, 105.

Lawson, C. L. \& Hanson, R. J. Solving Least Squares Problems (Prentice-Hall, Englewood Cliffs, New Jersey, 1974).

Lee, Y.-W. 1990, Ph.D. Thesis, Yale University.

Lee, Y.-W. 1993, these proceedings, pp. 285-294.

Lee, Y.-W., Demarque, P. \& Zinn, R. 1990, ApJ, 350, 155.

Liu, T. \& Janes, K.A. 1993, these proceedings, p. 28.

Longmore, A.J., Dixon,R., Skillen,I., Jameson,R. \& Fernley,J. 1990, MNRAS, 247, 684.

Longmore, A.J. 1993, these proceedings, pp. 19-27.

Mateo,M., Harris,H., Nemec,J., Olszewski,E. \& Schombert,J. 1988, BAAS, 20, 717.

Mateo, M., Harris, H.C., Nemec, J.M. \& Olszewski, E. 1990, AJ, 100, 469.

Nemec, J. M. In The Use of Pulsating Stars in Fundamental Problems of Astronomy, IAU Coll 111, (ed. E.G.Schmidt) pp. 215-245 (Cambridge University Press, 1989).

Nemec, J. M. \& Mateo, M. 1990, ASP Conf.Ser. 11, 64.

Nemec, J.M., Wehlau, A. \& Oliveira, C. 1988, AJ, 96, 528.

Petersen, J.O. 1993, A\&A, preprint.

Pike, C.D. \& Meston, C.J. 1977, MNRAS, 180, 613.

Sandage, A.R. 1970, ApJ, 162, 841.

Sandage, A.R.\& Katem, B. 1977, ApJ, 215, 62.

Sandage, A.R. 1990, ApJ, 350, 603.

Sandage, A.R., Katem, B. \& Sandage, M. 1981, ApJS, 46, 41.

Sawyer Hogg, H. 1973, PDDO, 3, 1.

Seber, G. A. F. 1977, Linear Regression Analysis, (Wiley, New York).

Simon, N. \& Lee, A.S. 1981, ApJ, 248, 291. 
Simon, N. \& Clement, C.M. 1993, these proceedings, pp. 304-312.

Smith, H.A., Silbermann, N.A., Baird, S.R. \& Graham, J.A. 1992, AJ, 104, 1430.

Walker, A. \& Mack, P. 1988, AJ 96, 1362.

Welch, D., Mateo, M., Olszewski, E., Fischer, P. \& Takamiya, M. 1993, AJ, 105, 146.

Zinn, R. 1985a, Mem.S.A.It., 56, 223.

Zinn, R. 1985b, ApJ, 293, 424.

Zinn, R. \& King, C.R. 1982, ApJ, 262, 700.

Zinn, R. \& West, M.J. 1984, ApJS, 55, 45.

\section{DISCUSSION}

J. MATTHEWS: In your current formulation, the slope of the $P-L$ relation is defined to be only a function of the type of variable star. What happens to your results, particularly for the anomalous Cepheids and Pop.II Cepheids, if the slope is also free to change from cluster to cluster?

J. NEMEC: If the stars of a given kind in a particular cluster are found to have a $P$ - $L$ slope different from that of the same kind of stars in another system, then one would have to think about the possibility that the stars are not of the same kind. Two relevant situations come to mind: (1) the $K$-band RR Lyrae photometry of L90 possibly suggests that the slope of the $P-L$ relation for the NGC 5466 RR Lyrae stars is steeper than that for the other RR Lyrae stars. Unfortunately the number of RR Lyrae stars in NGC 5466 is small $(\sim 20)$ and to check this preliminary result will require more accurate photometry of the available stars; (2) the two brightest Carina 'SX Phe stars' (Nemec \& Mateo 1989) appear to lie well above the $P-L$ relation for first-overtone SX Phe stars. Possibly these are higher-overtone pulsators, or a new kind of SX Phe star.

J.O. PETERSEN: Please comment on determination of absolute distances. In two to three years the HIPPARCOS satellite will provide an accurate parallax for SX Phe, and this information will be valuable for determining absolute distances from your relative distances. Is that correct?

J. NEMEC: Having tied our distance estimates to the zero-point cluster M15 the absolute distances depend directly on M15's assumed distance, which at present depends on Pop. II subdwarfs and the application of Lutz-Kelker corrections to the M15 main sequence. The HIPPARCOS results for SX Phe will certainly provide a much improved zero-point for all the $P-L-[\mathrm{Fe} / \mathrm{H}]$ relations discussed above, and they are eagerly awaited.

N. SIMON: The LNA models (but with old opacities) for short period ( $<3$ days) Type II Cepheids show that these stars lie in a region of the HR-diagram where the $\mathrm{F}$ and $\mathrm{H}$ blue edges have already crossed. This probably means that there ought not to be any overtone pulsators among these objects. 
A. COX: I suggest that your Pop. II Cepheid dichotomy is due to a factor of two in mass (coalescence) rather than pulsation mode. This would give $\sqrt{2}$ in your period ratio and would give your two sequences.

J. NEMEC: I believe Prof. Böhm-Vitense has something to say about your comment and about Prof. Simon's comment. Also, Dr. Moskalik has shown me earlier today a preprint of his that argues for the existence of Pop. II Cepheids pulsating in the first-overtone mode.

E. BÖHM-VITENSE: I think you can have overtone pulsators in regions of the HRdiagram where fundamental mode pulsations are also unstable. I do not know whether there are mass differences in the variables that Jim has studied. Since the lower luminosities occur for the longer periods you would need systematically lower masses for the longer period ones as compared to the shorter period ones. If the anomalous Cepheids are all merged stars then all will have the higher mass and you will not have two mass sequences.

A. SANDAGE: The fit of all horizontal branch levels to be the same, independent of $[\mathrm{Fe} / \mathrm{H}]$, poses a problem doesn't it? What is the range of $[\mathrm{Fe} / \mathrm{H}]$ of the clusters you used? If $\Delta M_{V}=0.3 \Delta[\mathrm{Fe} / \mathrm{H}]$, what is the error in the placement of the Type II Cepheids? Can this wipe out your two fundamental and first-overtone lines in the composite $P$ - $L$ relation?

J. NEMEC: Our first models (e.g., Fig. 2) assumed no dependence of HB-luminosity on metallicity, but the $P-L$ relations were still surprisingly tight because metal abundance acts like reddening - an incorrect value translates into an incorrect distance but does not introduce scatter into the diagram. Our subsequent models all assumed metallicity dependences (at $P=0.5$ day) of $M_{V}(\mathrm{RR} a b,-0.3)=0.32[\mathrm{Fe} / \mathrm{H}]+1.19$ (L90) and $M_{B}(\mathrm{RR} a b,-0.3)=0.35[\mathrm{Fe} / \mathrm{H}]+1.53$, and dealt with stellar evolution according to the recent models of Y.-W. Lee. The metallicity range of the stellar systems that contain RR Lyrae stars discussed here is from -2.5 (NGC 5053) to -0.99 (M107); hence, metallicity differences and the effects of stellar evolution are generally rather small.

D. WELCH: The real acid test for your fundamental/first-overtone sequences will be the $K$-photometry or the use of a colour to remove the temperature-induced scatter.

J. NEMEC: Since March 1992 we have had a program underway at the Apache Point Observatory in New Mexico to obtain $B, V, K$ photometry of the Cepheids in several galactic globular clusters. 